\title{
An Ontology-based Model for Urban Planning Communication
}

\author{
Draft version \\ Claudine Métral $^{1}$, Gilles Falquet ${ }^{2}$, Mathieu Vonlanthen ${ }^{2}$ \\ ${ }^{1}$ Institut d'architecture (IAUG), University of Geneva \\ 7, route de Drize, $\mathrm{CH} 1227$ Carouge, Switzerland \\ claudine.metral@archi.unige.ch \\ ${ }^{2}$ Centre universitaire d'informatique (CUI), University of Geneva \\ 24, rue Général-Dufour, CH 1204 Geneva, Switzerland \\ \{gilles.falquet, mathieu.vonlanthen\}@cui.unige.ch ${ }^{1}$
}

\begin{abstract}
Urban planning projects are complex and involve multiple actors ranging from urban planners to inhabitants. These actors differ greatly in their background or their centres of interest. The main objective of our research is contributing to a better communication of urban planning projects between the various actors involved. With this intention, we defined an ontology-based model whose main characteristics are, on the one hand, the semantic integration in a knowledge base of the urban knowledge coming from various sources such as GIS databases, master plans, local plans or any other document and, on the other hand, the modelling of the centre of interest of an urban actor. This models can then be used to generate adapted user interfaces to present the project's data and knowledge according to each actor's background and interests.
\end{abstract}

Keywords. Urban planning, ontology, knowledge base, semantic integration, 3D city model

\section{Introduction}

Urban planning is a complex process involving many actors, such as urban planners, inhabitants, employees of urban technical departments, or politicians, and broad range of interests and demands. For instance, inhabitants are more and more implied in the way their city, and hence their way of life, is intended to change. But communication between the different actors is not easy. For example a plan, which is an obvious tool for an urban planner, may be hard to understand for the general public. Conversely, tri-dimensional (3D) representations are usually preferred by non-specialists, but are seldom used by urban planners. Moreover urban actors naturally use different terms and are interested by different types of information. For example a textual technical

\footnotetext{
${ }^{1}$ This research has been funded by the swiss Secrétariat d'Etat à l'Education et à la Recherche as part of the COST C21 action.
} 
report with technical vocabulary is useful for an urban technical employee but not for a politician or an inhabitant who will probably misunderstand it. Finally, the actors are not necessarily interested in all aspects of a project and they may be interested in different aspects at different times. For instance, an actor may want to explore elements of a project that are related to water management, then he or she may be interested in data related to the safety on streets.

Thus, an efficient communication tool for urban planning projects must provide each actor with the information that is relevant for him or her and present this information in a way that is easily understood. This implies that the tool must take into account the user profiles and their centres of interest to present different views on the project.

As a contribution to improve the communication between the various actors involved in urban planning projects, we propose in this paper an ontology-based model that can serve as a basis to develop computerized tools for exploring and understanding urban projects. This model has two main components:

1. an integration component that is intended to represent in the same knowledge base information coming from different heterogeneous sources. This component is build around an ontology of urban planning process

2. an adaptable interface component whose aim is to provide each actor with a view of the urban project the corresponds to his or her profile and centre of interest. This component includes actor specific ontologies (viewpoint ontologies) and an ontology of themes

This paper is organized as follows: in the next section we briefly present tools that are currently used to represent data and knowledge in urban planning projects; in section 3 we introduce the notion of ontology and its applications in the urban planning domain; section 4 introduces the information integration part of our model and section 5 presents the user interface part of the model; section 6 gives our conclusion and perspectives for further work.

\section{Urban Planning Knowledge and Tools}

Working on an urban planning project involves working with heterogeneous and disseminated information obtained from various sources. These sources can be geographic information systems, master plans or local plans, legal texts, regulations, and, more recently, 3D city models.

\subsection{Geographic Information Systems}

A geographic information system (GIS) is essentially an information system that is intended to manage geographically-referenced information. It is usually comprised of a database system that stores geometric entities and can perform geometry or topology-based search operations. In the last decades, a lot of GIS containing spatial urban data have been created such as the Système d'information du territoire genevois (SITG) in Geneva. Such systems are very useful for example to obtain information on 
a parcel (owner, building) or to visualize information on a map (cycle paths, parking places reserved for handicapped persons, polluted sites). But GIS generally provide information about what exists, they cannot be considered as planning tools.

\subsection{Master and local plans}

Master plans are legal tools for the global planning of the territory. In Geneva the Cantonal Master Plan (Plan directeur cantonal) is the outcome of an extensive political and technical process to define in a consensual way the aspirations of the population concerning regional planning for the next fifteen years. Such a master plan is organised in different parts: texts, thematic maps (mobility, nature and landscape, etc.) and a synthesis map. The Cantonal Master Plan is available on the official web site of the State of Geneva. But, due to its density and its scale (the whole State of Geneva), it is difficult to retrieve precise information in it.

Local plans are legal tools for the planning of urban area under development or mutation. In Geneva they are subject to a public consultation. They are usually composed of texts and plans sometimes enriched with views and sections. If plans are usual tools for urban planners it is not true for the general public who often feels more at ease with 3D representations. Moreover local plans are not organized in the same way as the cantonal master plan, making difficult the crossing of information between master and local plans.

\subsection{D City Models}

3D representations of urban data are named 3D city models. Different projects that model an existing city have been developed or are under development around the world. They are intended for a wide range of applications, such as planning and design, infrastructures and facility services, marketing or promotion [10].

3D city models can be built from existing GIS, which contain basically 2D information. For example by combining and extruding different SITG information layers, such as the digital terrain model (representing the ground without the vegetation or the buildings), the building footprints and the building heights, we obtain a 3D block model of Geneva. Strictly speaking such a model where the third dimension is expanded from $2 \mathrm{D}$ data using heights is a $2.5 \mathrm{D}$ model, but we refer to it as a $3 \mathrm{D}$ city model.

By adding a texture mapping from the orthophotos of the area we obtain a more realistic 3D model. More generally 3D city models differ by elements such as their degree of reality, i.e. the amount of geometric details that are represented within them, their data acquiring methods and their functionality, i.e. the degree of utility and analytical features that they allow [10]. 


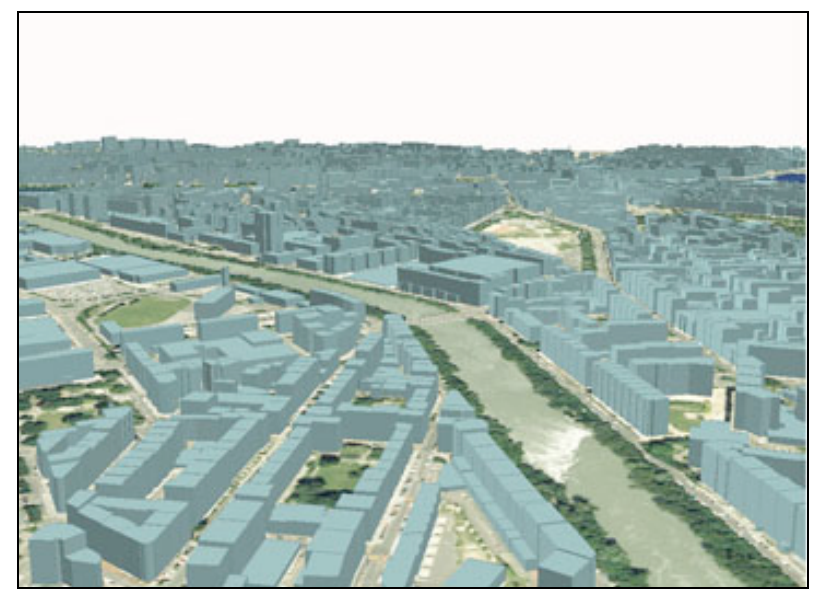

Fig. 1. 3D city model extracted from the SITG

CityGML [7] is a unified model for the representation of 3D city models based on the standard GML3 of the Open Geospatial Consortium. Urban objects (relief, buildings, vegetation, water bodies, transportation facilities, city furniture) are represented in CityGML by features with geometric, topological and thematic properties. CityGML ensures spatial consistency between 3D models at different scales (five levels of detail are possible).

$3 \mathrm{D}$ city models are useful for the visualization of full urban environments including built and natural structures or for the simulation of new urban projects with their environmental and visual impact. They are also a visual communication tool much more efficient than, for example, official plans. But what they represent is essentially of geometric nature when a lot of urban knowledge do not correspond to geometric entities (building period, parcel owner, building permit for example). Moreover 3D city models are not directly linked to the texts that are the main part of the master and local plans.

\section{Proposal for an Ontology-based Model for Urban Planning Communication}

An efficient communication of urban planning projects must, on the one hand, integrate the whole of the urban knowledge resulting from the various sources that are GIS databases, master plans, local plans, or any other document and, on the other hand, take in account the centres of interest of the different users. A centre of interest is in fact defined by a theme (mobility, environment, etc.) and the viewpoint that the user wants to have on this theme. Both the semantic integration of the urban knowledge and the specification of a user's centre of interest can't be based only on terms. The underlying semantics must be taken into account because some data and documents can be semantically related without containing the same terms. From where the idea to use ontologies. 


\subsection{Ontologies}

In the field of Artificial Intelligence several definitions of the term "ontology" have been given. According to Gruber an ontology is an "explicit specification of a conceptualization" [6]. A slightly different definition is "a formal, explicit specification of a shared conceptualisation" [13]. A conceptualization is an abstract, simplified view of some domain that we wish to represent for some purpose, i.e. the objects, concepts and other entities that are assumed to exist in some area of interest and the relationships that hold among them. "Formal" means that some formal representation language has been used and so that the ontology is machine-readable and machine-processable. "Explicit" means that both the type of concepts used and the constraints on their use have been defined [2]. "Shared" refers to a common understanding of some domain that can be communicated across people and computers [13]. Three important areas where ontologies could be used have been reported: communication between people with different needs and viewpoints, interoperability between heterogeneous systems and systems engineering [15].

\subsection{Urban Planning Ontologies}

The urban planning field is concerned with ontologies. In Urban Civil Engineering, some preliminary experiments of the Towntology project have been made, producing ontologies such as an ontology for street planning and mobility [14]. Another project is related to buried urban infrastructure with as main focus the routing/alignment of an infrastructure network in such a way that minimizes its conflicts with other systems [9].

\subsection{Ontology-based Communication of Urban Planning Projects}

From our part, we use an ontology approach both for integrating the different data and documents related to urban planning projects and for enabling a user to define the best interface that fits his requirements and wishes.

Section 4 describes the global ontology used for the semantic integration of the data and documents. Section 5 describes the various ontologies associated to the themes and the viewpoints. These ontologies are utilized by the users to select in the global ontology the knowledge that interests them. Section 6 describes the interface which main characteristics is its adaptability to the user.

\section{Semantic Integration of the Urban Knowledge}

One of the aims of our approach is to provide the user with an integrated view of an urban project. As we have seen above, the information about an urban project is represented in different media (databases, documents, 3D city models, etc.) and at different scales (from local plans to master plans). To integrate these information sources we propose to use an ontology of the urban planning project domain (OUPP) 
as a common conceptual reference and to connect the information sources to this ontology. We will thus obtain a knowledge base that represents the urban project.

This knowledge base is composed of:

- a conceptual layer (the OUPP) that describes all the concepts that appear in the documents and in the GIS database;

- a factual knowledge layer that links these concepts to the information sources.

There are two kinds of links between the information sources and the knowledge base.

- a conceptual annotation link connects an information element (a document or a part of a document) to a concept because this element mentions or is about this concept. For instance, a document about transportation would probably be connected to concepts such as "road", "street", "bus";

- an instance link indicates that an information element is a particular instance of a concept. For instance, a given local plan document can refer to parcels 1807 and 1809. In this case the link must contain some way to identify the instance (here the parcel number) and to find it in the information source (for instance with a database query or an XML pointer to a document element, etc.) .

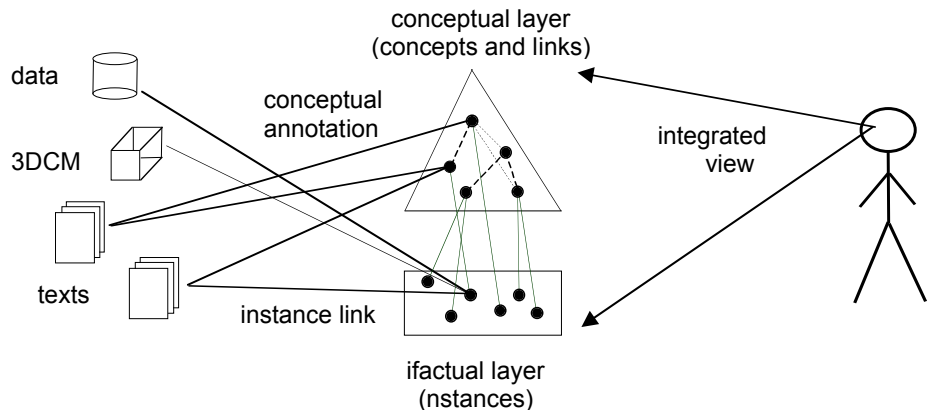

Fig. 2. Semantic integration of the urban knowledge

\subsection{Ontology Construction}

Research and practice in the field of ontologies showed that the construction of an ontology is a complex task requiring not only a great knowledge of the field to be described but also a control of the structuring of the concepts using formal languages. During the last years several approaches and tools have been developed to do these concept extractions automatically or semi-automatically. For instance [12] and [1] propose techniques to extract ontologies from relational database schemas, while [16] use text analysis technique to help in the construction of ontologies. At the same time, several languages have been developed to formalize ontologies, those being based primarily on predicate logic, on frames or on descriptive logic. The most recent works concern the language OWL which is a recommendation of the consortium W3C within the framework of the "semantic Web". New tools and new methods for analysis of ontologies are under development [4]. 
We have build the first version of the OUPP with a simple graph editor then we have formalized it with OWL-DL. We have reused some parts of urban ontologies developed in the framework of the Towntology COST action (the Ville and Transports ontologies). We have establish relationships to two of the main themes ("urban area", "mobility" and "rural area") of the Cantonal Master Plan of Geneva (Ville has been related to "urban area" and Transports to "mobility"). Then we have added concepts from the master and local plans and then from the SITG whose concepts are less general. For the moment two kinds of semantic relations have been defined: the "isA" relation and the "isAssociatedWith" relation, which is symmetric and transitive.

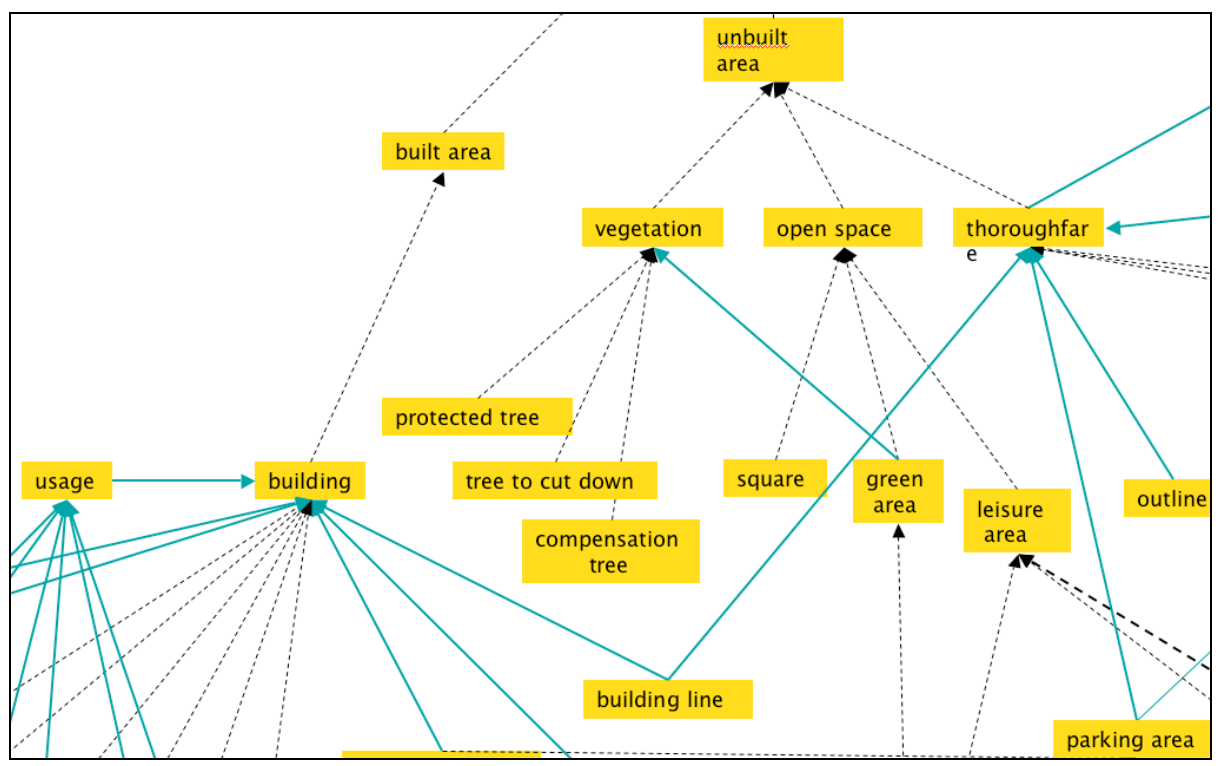

Fig. 3. Partial view of the OUPP.

Dotted lines represent isA links, solid lines represent isAssociatedWith links.

\subsection{Construction of the Factual Layer}

Knowledge can be defined as organized information which can be used to produce new meanings and generate new data. According to Sowa [11] a knowledge base is an informal term for a collection of information that includes an ontology as one component. In our case, the construction of the knowledge base consists in linking the collected data (GIS data, natural language texts, and plans) to the ontology. The construction of the knowledge base corresponds to a semantic integration. For the GIS database [3] the concepts and relations of the ontology are directly inferred from the GIS database schema, So linking concepts and instances is immediate. For natural language texts, the linking problem is much harder. Concept extraction can be done using the terms associated with each concept. The main problem is as always in 
natural language processing (NLP) the polysemic terms. There exists a lot of different disambiguation techniques [8] but evaluating the different techniques and selecting the most appropriate is beyond the scope of this article. Instances extraction can be done readily when we there exist object identifiers like local plan number or a proprietary name. But when such information is not available and when the instance is defined with natural language like in the example "the house near the river and beyond the hill" advanced NLP techniques are needed. The natural language text will be fragmented using the already available decomposition into chapter and paragraphs. Each fragment will be associated with concepts and instances extracted from it. For plans in vector representation, concepts are extracted from the key label and the whole plan is associated with the concept. Each symbol on the plan corresponding to the key label is associated with an instance of the concept. Moreover each instance has to be associated with a geographic position. Each time a new document is added into the system, concept and instance linking has to be done.

\section{An Ontology-Based Adaptable Interface}

\subsection{Viewpoints}

The actors involved in an urban planning process differ in many respects. In particular, they have different knowledge backgrounds and they use different vocabularies. To take account of this diversity we propose to represent it by different ontologies that correspond to the different types of actors. Each such ontology, called a viewpoint, represents the urban planning domain (or a part of it) as viewed by a given type of actor. It may of course differ from the OUPP in several aspects such as the terminology or the conceptual structure.

The idea is to use these viewpoints at the user interface level to:

- provide an interface that "speaks the user's language", i.e. all the interface elements such as menus, labels, etc. should use the user's own vocabulary;

- provide navigation tools that the user immediately recognizes;

- display the information elements according to the norms, conventions or usage of this category of users.

To reach this goal, it is necessary to connect each viewpoint-ontology to the information sources. This is accomplished by establishing alignment links between each viewpoint and the reference ontology of urban planning process, as shown on the figure below. The alignment links interconnect the concepts of two different ontologies through an equivalence or a subconcept relationship.

The OUPP acts here as a "pivot language" among the different viewpoints. The benefit of this knowledge organization is that the links (factual layer) between the information sources and the OUPP are established only once and serve for all the viewpoints. 


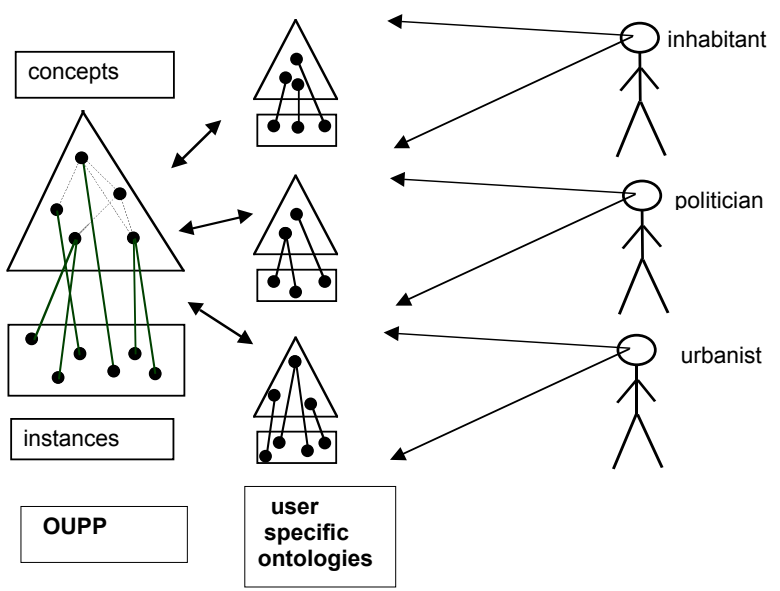

Fig. 4. Adaptation to user viewpoints

\subsection{Themes}

Within his or her own viewpoint, a user may be interested in different thematic aspects such as "transportation", "safety", "noise", etc. These themes, or subdomains, correspond to sets of concepts and links in an ontology that do not necessarily form a connected subgraph of the ontology structure. Since these themes are, for the most part, common to all actors, we can represent them in a common ontology of themes. The concepts of this ontology can then serve to index the concepts and links of the OUPP. Hence, a theme $T$ within the OUPP is made of all the concepts and links indexed by $T$.

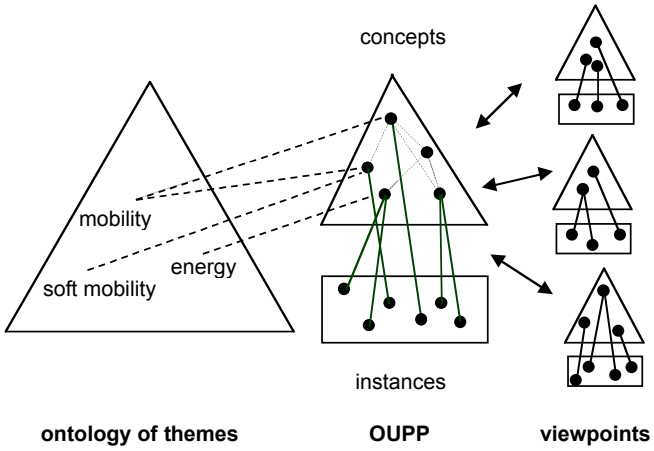

Fig. 5. Viewpoints and Themes

At the interface level, the themes will serve as filters. By choosing a theme the user will restrict his or her view to the information elements that are relevant for this theme. Thus he or she will be able to concentrate on this theme without being disturbed by irrelevant information. 


\subsection{Adapted visualization}

Ontologies have already been used for generating ontology-based interfaces. Among research works we can quote those realised at the University of Geneva [5] which generate hyperdocuments fitting the reading objectives or specific viewpoints of readers.

The ontology-based model we propose here is well suited to create adapted views of an urban planning project. This adaptation is based on the user profile, which is used to select a viewpoint-ontology $V$, and his or her current centre of interest, which corresponds to a theme $T$ in the ontology of themes. Following the virtual document approach, the interface composition itself proceeds in two steps:

1. $V$ and $T$ determine the concepts that are relevant for the user and should appear on the interface. In addition, $V$ provides the vocabulary that the interface must use for displaying information and for interface elements such as menus, lists of concepts, etc.

2. The conceptual annotation links and the instance links, together with the relations found in $V$ and in OUPP give rise to visual and hypertext links between interface objects.

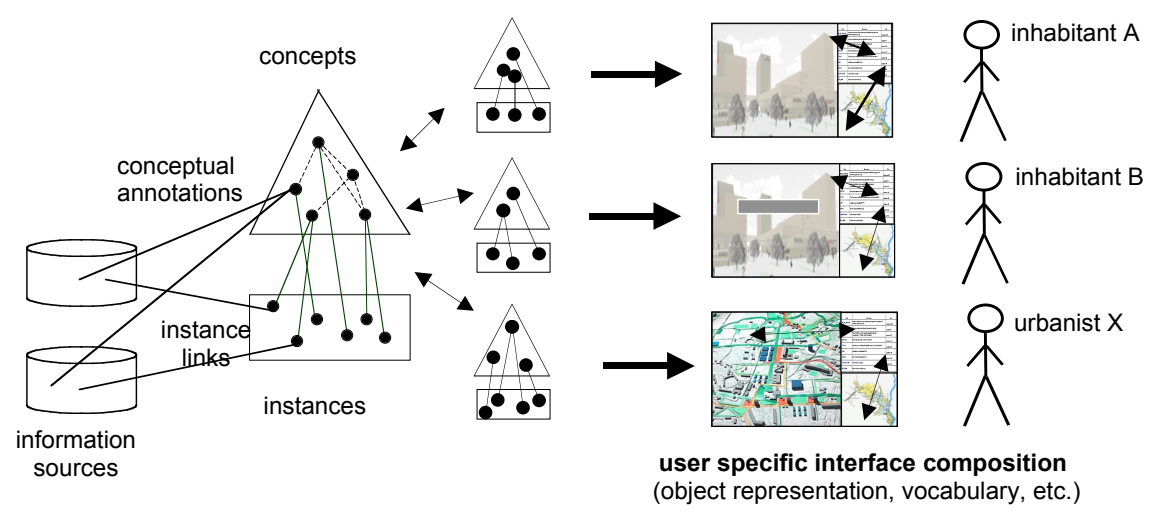

Fig. 6. Ontology-based user specific interfaces

The generation of links in the interface is carried out according to generation rules that correspond to different linking semantics. Typical rules are:

same instance: if the information elements $a$ and $b$ (belonging to different information sources) are both connected through an instance link to the same instance $x$ of a concept $c$, then generate a link between the visual representations of $a$ and $b$. This type of link is exemplified in figure 6 below, where the same object (building 107a) appears in a $3 \mathrm{D}$ view, on a plan and in a textual document. 


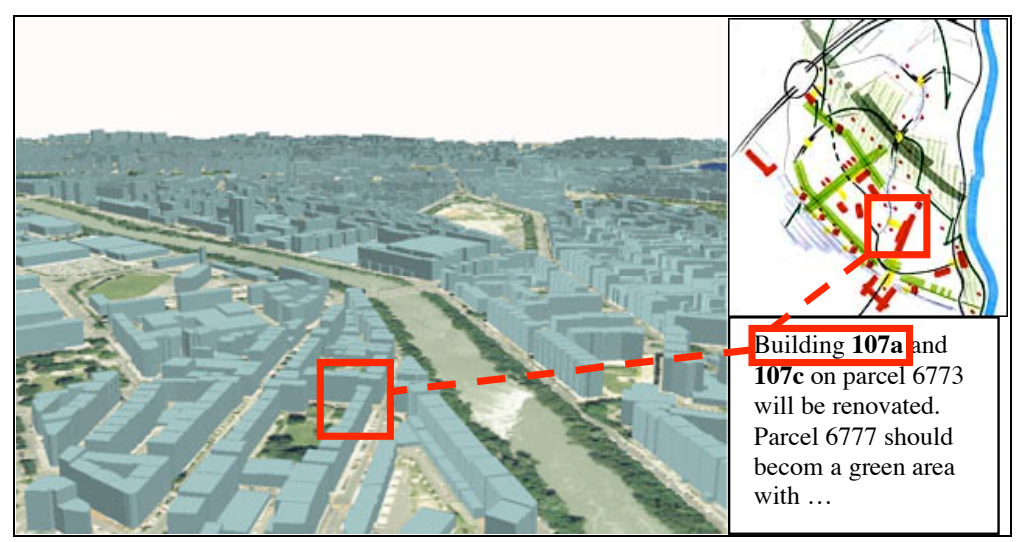

Fig. 7. Ontology-based links in the interface. The solid rectangles visually interconnect representations of the same building in different views.

instance to concept: this is a type of link connecting the representation of an instance to its definition in the ontology $V$ or to the definition of a related concept. For example a visualization related to "pedestrian path" can display not only such paths in the area of interest of the user but also the definition of "soft mobility" which is related to the concepts "pedestrian path" or "green path".

More complex rules may represent more sophisticated inferences involving the traversal of longer path in the knowledge base.

\section{Conclusions and Future Work}

In this paper we have presented a model that integrates in a knowledge base information and data from sources such as GIS databases, master plans, local plans or any document that seems to be relevant for the communication of an urban planning project. In addition to this semantic integration we specified an interface that fits a users's center of interest. We use an ontology-based approach both to the semantic integration and to the specification of the user interface.

The knowledge selected by the user by means of the ontology can be of various kinds: texts, plans, entities from the GIS database, etc. This knowledge is represented in our model as virtual documents that can take different forms ranging from hypertexts to $3 \mathrm{D}$ city models. However, there is still work to do, especially in finding and testing the best ways to represent non-geometric urban knowledge and linking documents with $3 \mathrm{D}$ city models. We also have to evaluate various interfaces with different urban actors and consequently to improve these interfaces according to their feedbacks. 


\section{References}

1. Astrova, I. (2004). Reverse Engineering of Relational Databases to Ontologies, In: Proceedings of the 1st European Semantic Web Symposium, LNCS 3053, 327-341.

2. Benjamins, V. R., Fensel, D. \& Gomez-Perez, A. (1998). Knowledge Management through Ontologies. In: Second International Conference on Practical Aspects of Knowledge Management, Basel, Switzerland.

3. Burrough, P.A. and McDonnell, R.A. (1998). Principles of Geographical Information Systems, Oxford University Press, Oxford.

4. Corcho, O., Fernández-López, M., Gómez-Pérez, A. (2003). Methodologies, Tools and Languages for Building Ontologies: Where is their Meeting Point? In: Data and Knowledge Engineering, 46(1), 41-64.

5. Falquet, G., Mottaz, C.-L., Ziswiler, J.-C. (2004). Ontology Based Interfaces to Access a Library of Virtual Hyperbooks. In: Rachel Heery, Liz Lyon (Eds.) Research and Advanced Technology for Digital Libraries. Proceeding of the 8th European Conference on Digital Libraries (ECDL 2004), Bath, UK, September 12-17, 2004. Lecture Notes in Computer Sciences (LNCS), vol. 3232, Springer, Berlin, Germany.

6. Gruber, T. R. (1993). A Translation Approach to Portable Ontology Specifications. In: Knowledge Acquisition 5(2), 199-220.

7. Kolbe, T. H., Gröger, G., Plümer, L. (2005). CityGML - Interoperable Access to 3D City Models. In: Proceedings of the Int. Symposium on Geo-information for Disaster Management, Delft, March 21-23.

8. Manning, C. D. and Schütze, H. (1999). Foundations of Statistical Natural Language Processing. MIT Press, Cambridge, MA.

9. Osman, H. (2004). A Knowledge-Enabled System for Coordinating the Design of CoLocated Urban Infrastructure. Research Summary. Department of Civil Engineering, University of Toronto.

10.Shiode, N. (2001). 3D Urban Models: Recent Developments in the Digital Modelling of Urban Environments in Three-dimensions. In: GeoJournal 52 (3), 263-269.

11.Sowa, J. F. (2000). Knowledge Representation: Logical, Philosophical, and Computational Foundations, Brooks Cole Publishing Co., Pacific Grove, CA.

12.Stojanovic, L., Stojanovic, N., Volz, R. (2002). Migrating Data Intensive Web Sites into the Semantic Web, In: Proceedings of the 17th ACM Symposium on Applied Computing, 11001107.

13.Studer, R., Benjamins, V. R. \& Fensel, D. (1998). Knowledge Engineering: Principles and Methods. In: Data and Knowledge Engineering, 25(1-2), 161-197.

14.Teller, J., Keita, A. K., Roussey, C., Laurini, R. (2005). Urban Ontologies for an Improved Communication in Urban Civil Engineering Projects. In: Proceedings of the International Conference on Spatial Analysis and GEOmatics, Research \& Developments, SAGEO 2005, Avignon, France, June 20th-23rd.

15.Uschold, M. \& Gruninger, M. (1996). Ontologies: Principles, Methods and Applications. In: Knowledge Engineering Review, 11(2), 93-155.

16.Velardi, P., Fabriani, P., Missikoff, M. (2001). Using Text Processing Techniques to Automatically Enrich a Domain Ontology. In: Proceedings of ACM FOIS, Ogunquit, Maine, USA, 270-284. 